\title{
Array-fed Partially Reflective Surface Antenna with Dynamic Beamwidth Control and Beam-Steering
}

\author{
Tomislav Debogovic, Juraj Bartolić \\ Department of Wireless Communications \\ University of Zagreb \\ Zagreb, Croatia \\ tomislav.debogovic@fer.hr
}

\author{
Julien Perruisseau-Carrier \\ Laboratory of Electromagnetics and Acoustics (LEMA) \\ EPFL \\ Lausanne, Switzerland \\ julien.perruisseau-carrier@epfl.ch
}

\begin{abstract}
This paper presents an approach to simultaneous beamwidth and beam-steering control using an array-fed reconfigurable partially reflective surface (PRS) antenna. Requirements and trade-offs regarding the design of such antennas are discussed. The antenna operation is confirmed by full-wave simulations.
\end{abstract}

Keywords-PRS antenna; Fabry-Pérot antenna; beamwidth; reconfiguration; phased array; beam-steering

\section{INTRODUCTION}

Partially reflective surface (PRS) antennas have attracted a significant attention due to their compact structure and high directivity [1]-[5]. The basic structure of such antennas is rather simple. A source antenna, typically a microstrip patch or a dipole with a reflector, feeds a PRS located approximately half of a wavelength above it. The PRS and the ground plane form a cavity where the electromagnetic waves originating from the source experience multiple reflections. At a given frequency the waves partially transmitted through the PRS interfere constructively and a highly directive pencil-beam is obtained. The directivity of the PRS antenna depends strongly on the PRS reflectivity [2]:

$$
D=D_{\text {source }} \cdot \frac{1+R}{1-R}
$$

where $D_{\text {source }}$ is the directivity of the source antenna, and $R$ is the PRS reflection coefficient magnitude.

The authors recently introduced a concept of dynamic beamwidth control in PRS antennas where directivity and beamwidth are controlled by electronic reconfiguration of the PRS reflectivity [6]-[8]. This concept strives to be a valid alternative to existing solutions for beamwidth control based on a mechanical reconfiguration [9]-[11].

In addition to the above-mentioned single-element feeds for PRS antennas, it is also possible to use an antenna array as a feed [12], [13]. In these references the aim of the array $+P R S$ combination is array thinning - owing to the extra directivity provided by the PRS, a certain number of the array elements can be dismissed while total directivity is preserved. This in turn leads to a simpler beam-forming network construction and overall cost reduction. Potential drawback of such an approach is the directivity bandwidth reduction due to the frequency dependence of the extra directivity enabled by the PRS.

In this paper we extend the above-mentioned concept by utilizing a phased array as a feed for reconfigurable PRS antenna, which results in a simultaneous beamwidth control and beam-steering. To the authors' knowledge, this is the first study of this kind. In Section II the issues regarding the design of such antennas are discussed. In Section III the results of fullwave simulations are presented, confirming the intended antenna operation. Finally, the paper is concluded in Section IV.

\section{ARRAY-FED RECONFIGURABLE PRS ANTENNAS DESIGN ISSUES}

We begin this section by recalling some aspects of antenna array theory that are critical in the context of reconfigurable PRS antennas fed by arrays. The most important issues are secondary lobes suppression, directivity enhancement, and interelement coupling. Then, we discuss how the antenna design and PRS reflectivity state affect these parameters and point out the necessary trade-offs.

\section{A. Secondary lobes suppression}

Fig. 1a shows an $N$-element linear array with uniform amplitude and interelement spacing. Its array factor reads [14]

$$
\begin{gathered}
A F=\sum_{n=1}^{N} \exp (\mathrm{j}(n-1) \delta) \\
\text { with } \delta=-\frac{2 \pi}{\lambda} \cdot d_{e l} \cdot \sin (\theta)+\alpha_{e x},
\end{gathered}
$$

where $\theta$ is the angle noted in Fig. 1a, $d_{e l}$ is the interelement spacing, and $\alpha_{e x}$ is the excitation phase difference between the array elements. For the reasons that will be made clear later on, the case of a large interelement spacing is especially relevant here. As an illustration, Fig. 1b shows the array factor of a linear four elements array with interelement spacing $d_{e l}=\lambda$, for 
two excitation phase differences. Notably, when all the elements are fed in phase $\left(\alpha_{e x}=0^{\circ}\right)$, the main beam of the array factor points toward broadside $\theta=0^{\circ}$, and there are grating lobes pointing in the end-fire directions $\theta=-90^{\circ}$ and $\theta=90^{\circ}$. Of course, a directive embedded element pattern would help to suppress the grating lobes. Nevertheless, if the main beam of the array factor is steered away from broadside by applying some excitation phase difference, say $\alpha_{e x}=60^{\circ}$, the grating lobes are also steered in the same direction. Actually, they are steered 'faster' than the main lobe, as shown in Fig. 1b. Hence, at some point of steering, the grating lobe will point in the direction where the embedded element pattern is 'strong', resulting in a poor secondary lobes suppression.
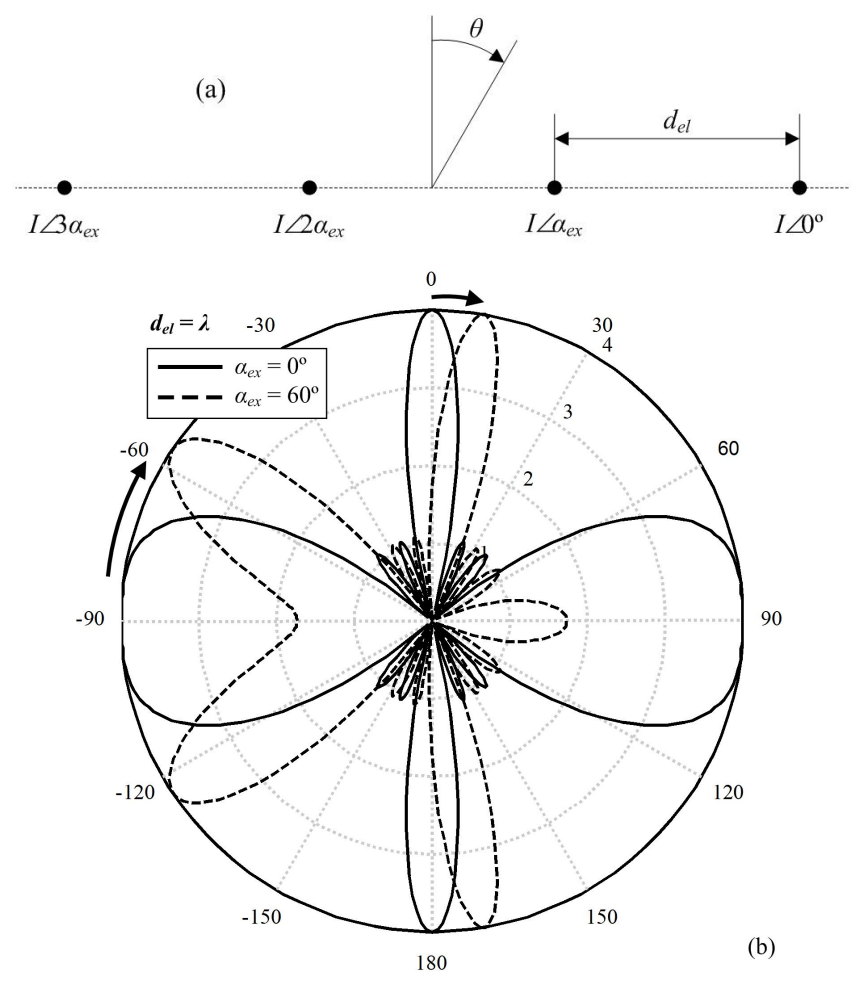

Figure 1. (a) Linear four elements array with uniform amplitude and interelement spacing; (b) Array factor of the four elements array with interelement spacing $d_{e l}=\lambda$.

\section{B. Directivity enhancement}

Another property of an antenna array that must be addressed here is directivity enhancement. Given that the coupling among the array elements is negligible, doubling the number of elements in a linear or planar array fed in phase doubles the directivity of the array. This rule stems from the definition of directivity [14]. However, it holds only if the array elements effective apertures do not intersect, as illustrated in Fig. 2a. If the array elements effective apertures do intersect, as shown in Fig. 2b, the directivity enhancement is less than expected, because the total effective aperture cannot be equal to a single array element effective aperture multiplied by the number of elements.

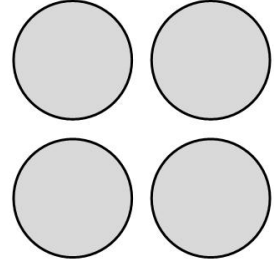

(a)

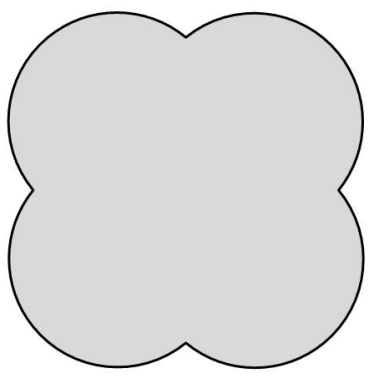

(b)
Figure 2. Directivity enhancement (the case of four elements planar array): (a) Element effective apertures do not intersect; (b) Element effective apertures intersect.

\section{Interelement coupling}

Coupling issues can be clarified by observing Fig. 3, which shows the array of four elements from the network point of view. Following the notations in Fig. 3, the input reflection coefficient at, say Port 1, reads

$$
\Gamma_{1}=S_{11}+S_{12} \cdot \exp \left(\mathrm{j} \alpha_{2}\right)+S_{13} \cdot \exp \left(\mathrm{j} \alpha_{3}\right)+S_{14} \cdot \exp \left(\mathrm{j} \alpha_{4}\right)
$$

where $\alpha_{2-4}$ are the excitation phases at Ports 2-4, taken relative to the excitation phase at Port 1. Therefore, the input impedance of any port generally depends on the other ports excitations. Since this complicates the design of a matching network, it is important to have as low coupling as possible. Then, all $S$-parameters representing the coupling $\left(S_{i j}, i \neq j\right)$ can be neglected, and reflection coefficient at port $i$ reads $\Gamma_{i} \approx S_{i i}$. Furthermore, in the case of arrays that operate in dual polarization, it is also important that all the $S$-parameters associated with coupling between the orthogonal ports are small. Otherwise, steering of the beam in one polarization would affect the beam direction in orthogonal polarization.

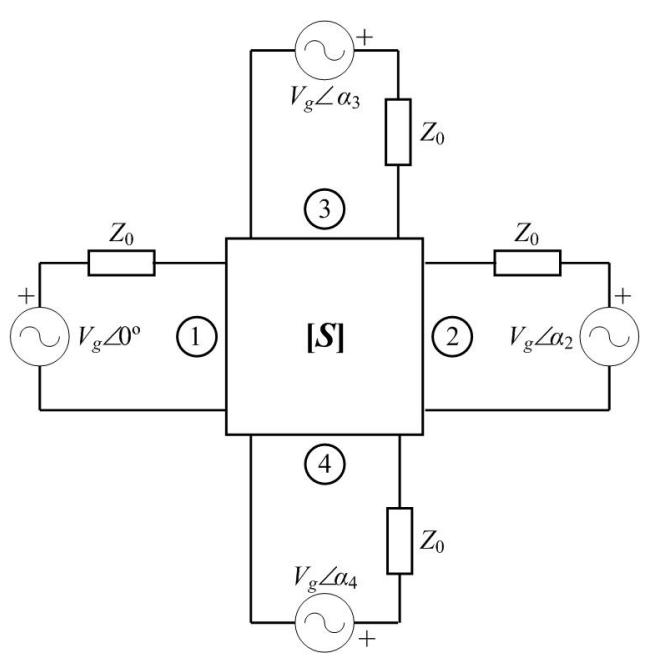

Figure 3. Four elements antenna array from the network point of view. 


\section{Discussion}

A reconfigurable PRS antenna fed by an array of microstrip patches is shown in Fig. 4. The effects introduced by a reconfigurable PRS above are very different in the cases of high and low state of the PRS reflectivity. The PRS effects can be best understood by taking a single patch together with a PRS above as an array element.

In the state of high PRS reflectivity, the array elements are already rather directive due to the high extra directivity enabled by the PRS. Thus, it is easy to achieve good secondary lobes suppression, even in the case of larger interelement spacing $d_{e l}$ and considerable beam-steering. However, coupling among the elements, caused by strong reflections from the PRS, is critical in this PRS state. In order to reduce it, the interelement spacing should be enlarged. In addition, a larger interelement spacing helps to improve the directivity enhancement because the array element effective apertures intersect less (see Fig. 2).

On the contrary, when the PRS reflectivity is low, the array elements should be close to each other. The main reason is that due to the lower directivity of the array elements, secondary lobes suppression is critical, especially when the beam is steered. Setting elements closer would prevent the appearance of grating lobes, or at least keep them in directions away from the broadside direction. At the same time, coupling is not a problem, because now the reflections from the PRS are weak. Furthermore, as the array elements are now less directive, they have smaller effective apertures and their cross-section is relatively small even if the interelement distance $d_{e l}$ is small.

Therefore, unfortunately the requirements on the interelement spacing $d_{e l}$ are completely opposite in the two extreme reflectivity states of a reconfigurable PRS. Since the antenna must be free of mechanically moving parts, interelement spacing must be constant, and compromises must be made. Nevertheless, these compromises still allow for acceptable results, as demonstrated in the following section.

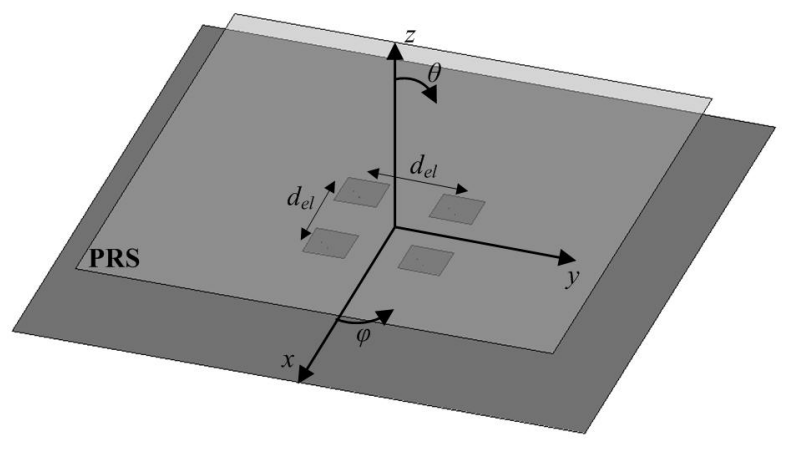

Figure 4. Structure of the array-fed reconfigurable PRS antenna.

\section{SIMULATION RESUltS}

The following simulation results were obtained in HFSS with an array of four microstrip patch antennas operating in dual polarization as the feed for a reconfigurable PRS antenna. The patches are arranged in a planar array, as shown in Fig. 5.
Two values of interelement spacing are considered, namely $d_{e l 1}=\lambda$, and $d_{e l 2}=1.2 \lambda$. The design frequency is $f_{0}=2 \mathrm{GHz}$. Also, two reconfigurable PRSs set above the patch array are considered. The 'equivalent' PRS models are employed since they have been proven to enable rather efficient full-wave simulations [7]. $\mathrm{PRS}_{1}$ has variable reactance in the range from -320 to $-130 \Omega$, and it is set at the distance $d=82.21 \mathrm{~mm}$ above the ground plane [6]. $\mathrm{PRS}_{2}$, set at the distance $d=80.57 \mathrm{~mm}$ above the ground plane, is more reflective in the high reflectivity state, having the reactance ranging from -320 to $-95 \Omega$. Both PRSs have modest ranges of reflectivity, which stems from the requests discussed in the previous section: for higher PRS reflectivity in the upper limit the coupling among the ports would be too high, while for lower PRS reflectivity in the lower limit the suppression of secondary lobes would not be sufficient. Finally, the size of both PRSs is equal to $5 \lambda \times 5 \lambda$ at the design frequency.

The most interesting results are listed in Tables 1 and 2 . Table 1 shows the results obtained for the antenna with $\mathrm{PRS}_{1}$ and $d_{e l 1}=\lambda$, while Table 2 shows the results for the antenna with $\mathrm{PRS}_{2}$ and $d_{e l 2}=1.2 \lambda$. In both tables the patch array under the PRS (Array $+P R S$ ) is compared to the single patch under the same PRS (Single patch $+P R S$ ) as the reference. The results obtained for the Array $+P R S$ confirm the discussion from the previous section. The directivity and beamwidth control is still present, although its range is somewhat smaller than the same range obtained for the corresponding single patch under the PRS. This is because in the high PRS reflectivity state the array elements effective areas (Single patch $+P R S)$ are large and intersecting. Thus, combining these elements in the array brings only about $1-3 \mathrm{~dB}$ of directivity enhancement, instead of expected $6 \mathrm{~dB}$. The enhancement is evidently larger for bigger interelement spacing and lower PRS reflectivity (see Table 2), since they both reduce the crosssection of the array elements effective apertures. In the low PRS reflectivity state the array elements effective areas are smaller and intersect less, which leads toward higher directivity enhancement of about 4-5 dB.

The main lobe can be steered in a limited range around the broadside direction. The criterion for determining the steering range is the secondary lobes suppression which has to be better or equal to $10 \mathrm{~dB}$. The beam-steering range is the result of compromises mentioned in the previous section. Due to the considerable interelement distance, high secondary lobes emerge quite soon when the beam is steered away from broadside. The results in Table 2 confirm this statement, since the obtained beam-steering range in the low PRS reflectivity state is smaller than in Table 1 due to the larger interelement distance.

Fig. 6 shows the interelement coupling for the antenna with higher reflectivity PRS and smaller interelement distance, which is the worst case considered in terms of coupling. Regarding the co-polarized ports, we found the coupling in $H$-plane to be the highest $\left(S_{51}\right)$. As for the cross-polarized ports, the most critical coupling is between the ports on the same $\operatorname{patch}\left(S_{21}\right)$. 


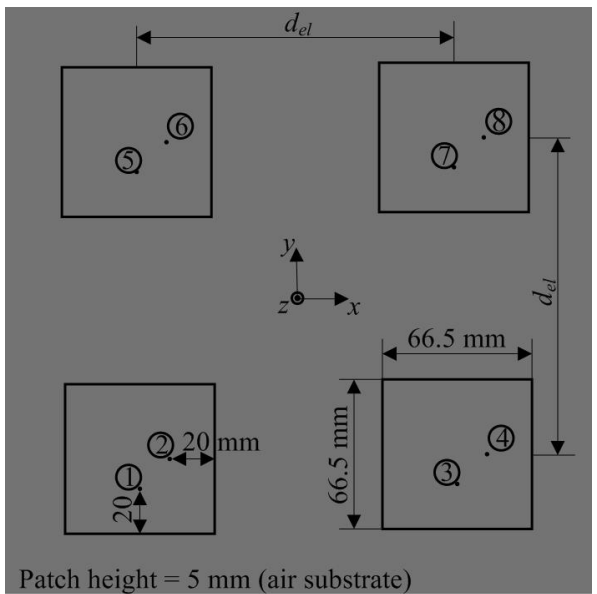

Figure 5. Microstrip patch array as the feed for a reconfigurable PRS antenna.

TABLE I. $\quad$ ANTENNA WITH PRS $\mathrm{P}_{1}$ AND $d_{e l 1}=\lambda$

\begin{tabular}{|c|c|c|c|c|c|}
\hline \multirow{2}{*}{\multicolumn{2}{|c|}{$\begin{array}{l}\text { Structure } \\
\text { PRS reactance }\end{array}$}} & \multicolumn{2}{|c|}{ Single patch $+P R S$} & \multicolumn{2}{|c|}{ Array $+P R S$} \\
\hline & & $-95 \Omega$ & $-320 \Omega$ & $-95 \Omega$ & $-320 \Omega$ \\
\hline \multicolumn{2}{|c|}{ Directivity } & $19.9 \mathrm{dBi}$ & $13.2 \mathrm{dBi}$ & $20.8 \mathrm{dBi}$ & $17.7 \mathrm{dBi}$ \\
\hline \multirow{2}{*}{$\begin{array}{l}\text { Beam- } \\
\text { width }\end{array}$} & $E$-plane & $15.1^{\circ}$ & $36.4^{\circ}$ & $14.1^{\circ}$ & $22.8^{\circ}$ \\
\hline & $H$-plane & $16.5^{\circ}$ & $39.3^{\circ}$ & $14.8^{\circ}$ & $24.0^{\circ}$ \\
\hline \multirow{2}{*}{$\begin{array}{l}\text { Beam- } \\
\text { steering }\end{array}$} & $E$-plane & \multirow{2}{*}{\multicolumn{2}{|c|}{ NA }} & $-6 \ldots+6^{\circ}$ & $-8 \ldots+6^{\circ}$ \\
\hline & $H$-plane & & & $-6 \ldots+6^{\circ}$ & $-7 \ldots+7^{\circ}$ \\
\hline
\end{tabular}

TABLE II. ANTENNA WITH PRS ${ }_{2}$ AND $d_{e l 2}=1.2 \lambda$

\begin{tabular}{|l|l|c|c|c|c|}
\hline Structure & \multicolumn{2}{|c|}{ Single patch + PRS } & \multicolumn{2}{c|}{ Array + PRS } \\
\hline PRS reactance & $-130 \Omega$ & $-320 \Omega$ & $-130 \Omega$ & $-320 \Omega$ \\
\hline Directivity & $18.0 \mathrm{dBi}$ & $13.4 \mathrm{dBi}$ & $20.7 \mathrm{dBi}$ & $18.5 \mathrm{dBi}$ \\
\hline \multirow{2}{*}{$\begin{array}{l}\text { Beam- } \\
\text { width }\end{array}$} & $E$-plane & $18.6^{\circ}$ & $35.9^{\circ}$ & $15.3^{\circ}$ & $20.1^{\circ}$ \\
\cline { 2 - 6 } & $H$-plane & $21.4^{\circ}$ & $39.8^{\circ}$ & $16.4^{\circ}$ & $21.2^{\circ}$ \\
\hline $\begin{array}{l}\text { Beam- } \\
\text { steering }\end{array}$ & $E$-plane & \multicolumn{2}{|c}{$\mathrm{NA}$} & $-7 \ldots+7^{\circ}$ & $-5 \ldots+4^{\circ}$ \\
\cline { 2 - 4 } \cline { 5 - 6 } & $H$-plane & \multicolumn{2}{|c}{} & $-7 \ldots+7^{\circ}$ & $-4 . .+4^{\circ}$ \\
\hline
\end{tabular}

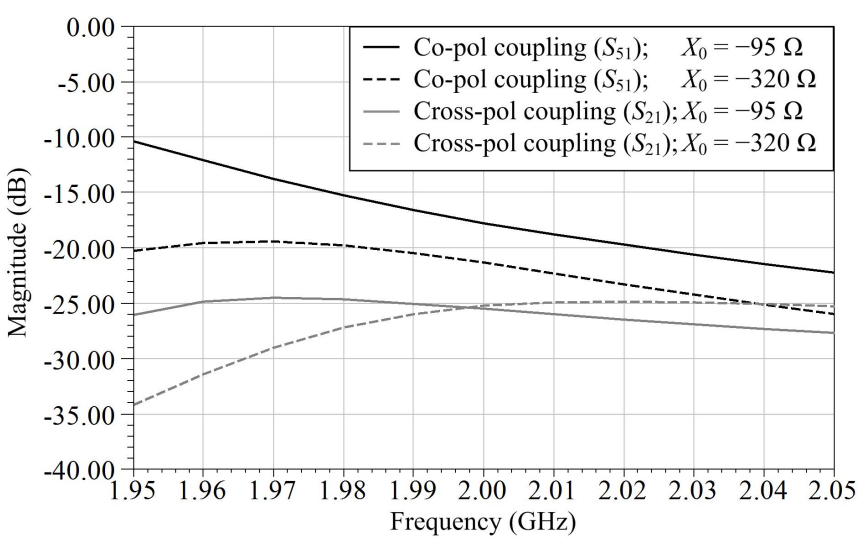

Figure 6. Interelement coupling in the worst considered case (antenna with PRS1 and $d_{e l 1}=\lambda$ ).

The results presented in Tables 1 and 2 were obtained by exciting only the odd-numbered ports of the microstrip patches. The even-numbered ports, which serve for exciting the antenna in the orthogonal polarization, were terminated by the system impedance of $50 \Omega$. However, it has been observed that the beamwidth control and beam-steering can be performed rather independently in two orthogonal polarizations, owing to the very low coupling between orthogonal ports.

Finally, Fig. 7 illustrates the dynamic beamwidth and beam-steering control for the combination of the $\mathrm{PRS}_{1}$ and interelement spacing $d_{e l 1}=\lambda$. The figure shows both beamsteering in $E$-plane and $H$-plane. In addition, the required excitation phases are stated for each shown result.
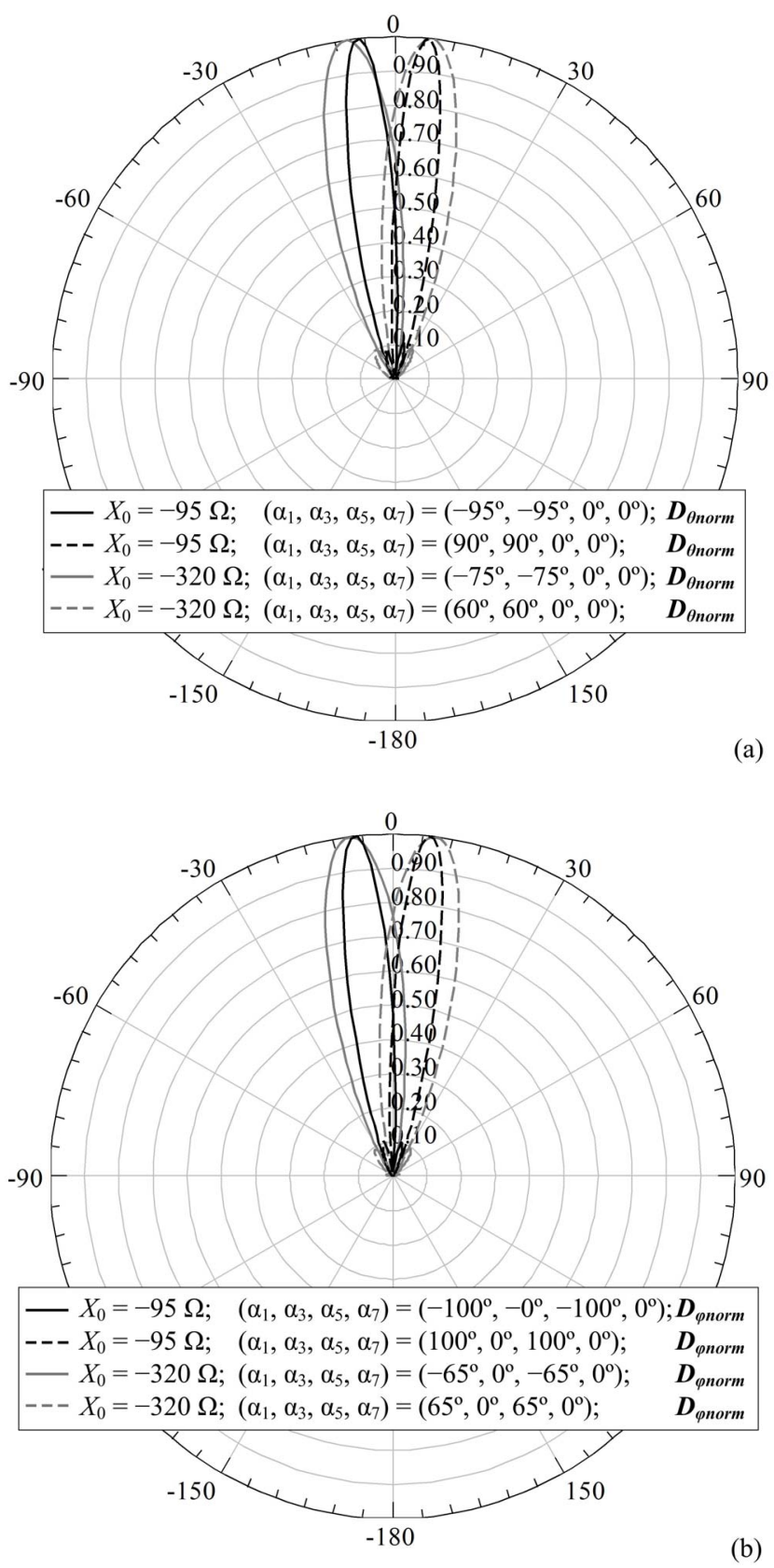

Figure 7. Dynamic beamwidth and beam-steering control (for the combination of the $\mathrm{PRS}_{1}$ and interelement spacing $d_{e l 1}=\lambda$ ): (a) E-plane; (b) $H$-plane. 


\section{CONCLUSION}

A novel approach to simultaneous beamwidth and beamsteering control, based on an array-fed reconfigurable PRS antenna, has been investigated. Essential design considerations were discussed and the antenna operation has been confirmed by full-wave simulations.

\section{ACKNOWLEDGMENT}

This work was supported in part by the FP7 European project "Coordinating Antenna Research in Europe" (CARE), the Swiss National Science Foundation (SNSF) under grant $\mathrm{n}^{\circ} 133583$, and the EU COST Action VISTA (IC1102).

\section{REFERENCES}

[1] G. von Trentini, "Partially reflective sheet arrays," IRE Trans. on Antennas and Propagation., vol. 4, no. 4, pp. 666-671, Oct. 1956.

[2] A. P. Feresidis and J. C. Vardaxoglou, "High gain planar antenna using optimised partially reflective surfaces," IEE Proc.-Microw. Antennas Propag., vol. 148, no. 6, pp. 345-350, Dec. 2001.

[3] A. P. Feresidis, G. Goussetis, S. Wang, and J. C. Vardaxoglou, "Artificial magnetic conductor surfaces and their application to lowprofile high-gain planar antennas," IEEE Trans. Antennas Propag., vol. 53, no. 1, pp. 209-215, Jan. 2005.

[4] N. Guerin, S. Enoch, G. Tayeb, P. Sabouroux, P. Vincent, and H. Legay, "A metallic Fabry-Perot directive antenna," IEEE Trans. Antennas Propag., vol. 54, no. 1, pp. 220-224, Jan. 2006.
[5] A. R. Weily, T. S. Bird, and Y. J. Guo, "A reconfigurable high-gain partially reflecting surface antenna," IEEE Trans. Antennas Propag., vol. 56, no. 11, pp. 3382-3390, Nov. 2008.

[6] T. Debogovic, J. Perruisseau-Carrier, and J. Bartolic, "Partially reflective surface antenna with dynamic beamwidth control," IEEE Antennas Wireless Prop. Lett., vol. 9, pp. 1157-1160, 2010.

[7] T. Debogovic, J. Perruisseau-Carrier, and J. Bartolic, "Equivalent surface modelling for reconfigurable partially reflective surface antennas," in Proc. 5th European Conference on Antennas and Propagation (EuCAP 2011), Rome, 2011.

[8] T. Debogovic, J. Bartolic, and J. Perruisseau-Carrier, "On the beamwidth control of partially reflective surface antennas," in Proc. $33^{\text {rd }}$ ESA Antenna Workshop, Noordwijk, 2011.

[9] A. D. Olver and J. U. I. Syed, "Variable beamwidth reflector antenna by feed defocusing," IEE Proc.-Microw. Antennas Propag., vol. 142, no. 5, pp. 394-398, Oct. 1995.

[10] H. Luh, "Variable beamwidth and zoom contour beam antenna systems," U.S. Patent 6414 646, July 2, 2002.

[11] Ph. Voisin, Ph. Ginestet, E. Tonello, and O. Maillet, "Payloads units for future telecommunication satellites - a Thales perspective," in Proc. $40^{\text {th }}$ European Microw. Conf., Paris, 2010, pp. 1786-1789.

[12] R. Gardelli, M. Albani, and F. Capolino, "Array thinning by using antennas in a Fabry-Perot cavity for gain enhancement," IEEE Trans. Antennas Propag., vol. 54, no. 7, pp. 1979-1990, July 2006.

[13] J. C. Iriarte, I. Ederra, R. Gonzalo, Y. Brand, A. Fourmault, Y. Demers, L. Salgetti-Drioli, and P. de Maagt, "EBG superstrate array configuration for the WAAS space segment," IEEE Trans. Antennas Propag., vol. 57, no. 1, pp. 81-93, Jan. 2009.

[14] C. A. Balanis, Antenna theory-Analysis and design (3 ${ }^{\text {rd }}$ edition). Hoboken, NJ: John Wiley \& Sons, 2005. 